\title{
SocArXiv
}

Journal of Criminal Justice Education : August 30, 2021

doi.org/10.1080/10511253.2022.2027477

\section{Jumpstarting the Justice Disciplines: A Computational-Qualitative Approach to Collecting and Analyzing Text and Image Data in Criminology and Criminal Justice Studies}

\author{
Alex Luscombe \\ University of Toronto
}

\author{
Jamie Duncan \\ University of Toronto
}

\author{
Kevin Walby \\ University of Winnipeg
}

\begin{abstract}
Computational methods are increasingly popular in criminal justice research. As more criminal justice data becomes available in big data and other digital formats, new means of embracing the computational turn are needed. In this article, we propose a framework for data collection and case sampling using computational methods, allowing researchers to conduct thick qualitative research - analyses concerned with the particularities of a social context or phenomenon - starting from big data, which is typically associated with thinner quantitative methods and the pursuit of generalizable findings. The approach begins by using open-source web scraping algorithms to collect content from a target website, online database, or comparable online source. Next, researchers use computational techniques from the field of natural language processing to explore themes and patterns in the larger data set. Based on these initial explorations, researchers algorithmically generate a subset of data for in-depth qualitative analysis. In this computationally driven process of data collection and case sampling, the larger corpus and subset are never entirely divorced, a feature we argue has implications for traditional qualitative research techniques and tenets. To illustrate this approach, we collect, subset, and analyze three years of news releases from the Royal Canadian Mounted Police website $(\mathrm{N}=13,637)$ using a mix of web scraping, natural language processing, and visual discourse analysis. To enhance the pedagogical value of our intervention and facilitate replication and secondary analysis, we make all data and code available online in the form of a detailed, step-by-step tutorial.
\end{abstract}

Keywords: computational social science, research methods, qualitative research, sampling, web scraping, criminology, criminal justice studies. 


\section{Introduction}

A growing body of literature in criminology and criminal justice focuses on the use of big data by criminal justice agencies or the potential for big data to reproduce or exacerbate existing patterns of inequality (Brayne 2017; Chan and Bennett Moses 2016; Ferguson 2017; HannahMoffat 2019). In some contexts, big data is poised to change policing and state surveillance in ways that expand and intensify social control (Joh 2016; Sanders and Sheptycki 2017). Less examined in extant literature are the ways that social researchers can methodologically benefit from working with large data sets to extend scholarly insight into practices of power, control, and sovereignty and related injustices.

In criminology and criminal justice studies, there have been calls for scholars to improve their engagement with the collection and use of big data and computational approaches to research (Smith et al. 2017). There are also longstanding concerns across the social sciences about a "coming crisis" and the limits of social science research (Savage and Burrows 2007; Gouldner 1972). These concerns are largely methodological and require research innovation to boost the trustworthiness, transparency, and credibility of social science disciplines. Criminology and criminal justice pedagogy similarly need an update. As Smith et al. (2017, 264) argue, "Criminological pedagogy... need incorporate computational methods training and its students and practitioners must become more digitally and algorithmically literate." In this article, we contend computational methods - especially when blended with traditional logics of qualitative inquiry - have the capacity to enrich the methodological program of criminology and criminal justice studies in ways that address concerns about methodological and pedagogical stagnation as well as trustworthiness, transparency, and credibility in the social sciences.

We propose a transferable framework for the blending of computational and qualitative methods in criminology and criminal justice studies. We illustrate this framework with a case study that can be replicated by readers who are interested in learning these methods in a more handson way. Readers interested in reproducing the case study illustration in this paper should consult the article supplement available at https://alexlusco.github.io/jsjd-supplement/. The code and tutorial-style instructions provided in the article supplement can also be used by readers that may wish to extend or adapt the data collection, manipulation, and sampling procedures used in this study for their own purposes. Readers may wish to use the material provided in the article supplement to simply replicate our procedures as well - to the extent possible $^{1}$ - as means of quality control. No previous experience in programming is required to follow along with the materials in the article supplement, though some background in the $\mathrm{R}$ programming language will be required to run and reproduce the code. At the beginning of the supplement, readers with little to no experience in the $\mathrm{R}$ programming language are directed to several free introductory books and tutorials where they can learn the requisite foundations. In addition to sharing code, we have provided detailed instructions at every step in the supplement. Consistent with our approach to "algorithmic thinking in the public interest" (Luscombe et al. 2021), the article supplement provides a completely transparent and relatable account of our methodological steps and decisions.

We begin by situating the use of computational and qualitative methods in context, partic-

\footnotetext{
${ }^{1}$ As this study is part computational, part qualitative, not all procedures can be easily replicated, nor, we would argue, should they be. While readers can easily replicate the data collection, manipulation, and sampling procedures used this in this study - and we would certainly encourage them to do so - other aspects of the research design, such as qualitative analysis of image data, should be evaluated in accordance with criteria for quality standard in qualitative research (Tracy 2010), which generally excludes replication as a goal.
} 
ularly in relation to the 'computational turn' in criminology and criminal justice studies. In part two, we introduce a transferable and adaptable framework for blending computational methods with qualitative research in a mixed-methods design. In part three, we demonstrate the application of this framework in a case study analysis of the images contained in police news releases in Canada. We collect, clean, explore, and sample text and image data computationally, and then analyze the images qualitatively using the software NVivo 12 (Jackson and Bazeley 2019). Whereas quantitative and qualitative logics are usually counter posed in academic literature, we work to coherently integrate them as a means of improving transparency and trustworthiness through a systematic and partially replicable approach to data collection and analysis. In the discussion and conclusion, we assess the implications of computational methodology for key concepts and procedures in qualitative inquiry.

\section{The Computational Turn in Criminology and Criminal Justice Studies}

Across the social sciences, scholars have made calls for research to capitalize on the potential of digital data. Savage (2015) and Smith et al. (2017) challenge social researchers to develop new digital research methods that enhance the conceptual, methodological, and empirical contributions of their disciplines. The emergence of 'big data' is viewed as a transformative development for social science (McKie and Ryan 2015). Halford and Savage (2017) contend use of big data allows scholars to generate knowledge about what organizations really are and how they operate. Computational methods can contribute to what Back $(2012,6)$ call 'live methods' by helping to "develop creative, public and novel modes of doing imaginative and critical sociological research."

Computational techniques for collecting and analyzing text and image data are becoming increasingly popular in criminology and criminal justice studies (Al-Zaidy et al. 2012; Khorshidi et al. 2021; Kleck et al. 2006; Kuang et al. 2017; Pina-Sánchez et al. 2019b,a; Snaphaan and Hardyns 2019; Vander Beken et al. 2021; Williams and Burnap 2016). Although much of the discussion and application of computational methods to date pertains to quantitative research, there is much to be gained from the advancement and application of computational techniques in support of qualitative research too. Computational techniques can offer a powerful means of data collection, data exploration, and sampling in qualitative research.

The term 'big data' is difficult to define, though many scholars agree on three attributes, referred to as the three Vs: volume, variety, and velocity (Kitchin 2014; McAfee et al. 2012). Volume refers to the amount of data, which can be in the millions or billions of observations; variety refers to the types of digital data now in existence, which vary enormously; and velocity refers to the unprecedented speed through which these data are created, updated, processed, merged, and made available. Researchers may alternatively opt to define big data in terms of the types of sources they use and how they access data, such as content from social media, rather than the amount of data per se (Cheng 2021). We define big data as the large-scale data that can be systematically accessed and analyzed by means of computational methods. These data are commonly too immense and multifaceted for traditional data-processing software like SPSS or Excel, requiring additional expertise in database management and local as well as cloud computing infrastructure to handle, and are too large to be handled in their entirety using qualitative methods. 'Large-scale' in this sense need not refer to data in the millions or billions. In this article, we begin from a corpus of over 13,000 police news releases, which certainly counts as 'big data' from a traditionally qualitative perspective. 
The benefits of developing computational approaches for social research are immense. Savage and Burrows (2007) advanced the idea of the "coming crisis of empirical sociology" to underscore the declining credibility and trustworthiness of certain traditional research methods such as phone-based surveys. They called on social researchers to update their methodological toolkits for the 21st century, an argument that applies equally to the justice disciplines (Smith et al. 2017). As more information goes online, a trend amplified in many ways by the COVID-19 pandemic (Lippert and Molnar ming), new techniques of online data collection and analysis are needed for the social sciences to stay relevant. Computational methods have long been popular among linguists and information scientists. Many social scientists, by contrast, are only now beginning to embrace the potential of computational techniques.

We are not arguing that computational methods are a replacement for in-depth, qualitative, interpretative approaches to exploring the meanings of social and cultural practices. Nor do we suggest that computational methods should or could replace established quantitative methods like online surveys or experiments. Computational approaches, like any methodological strategy (Travers 2009), have their strengths and weaknesses. Computational techniques need not be viewed as a standalone approach to data collection, but rather can be fruitfully combined with other methods in a mixed-methods design. Computational approaches offer a distinct methodological trajectory that can incorporate both qualitative and quantitative methods and logics of inquiry with potential to disrupt longstanding divisions between the two.

Tracy's (2010) criteria for quality in qualitative research offers a set of guidelines for ethically working with and interpreting information with sincerity, credibility, and rigour, and many of these can help guide projects that blend computational and qualitative methods. By collecting a much larger dataset over time, computational techniques can facilitate a research design that is longitudinal (Thomson and Holland 2003), something that can be difficult using traditional qualitative methods alone. As social scientists we design research projects that respond to doubts concerning existing theories (Locke et al. 2008) and craft our specific research questions based on gaps in the literature or problematization of specific phenomena (Sandberg and Alvesson 2011). We also seek forms of leverage in our data and analyses to ensure our interpretations and inferences are compelling (Collier et al. 2004). These and other demands central to social research can be addressed by blending computational and qualitative methods.

Many computational approaches rely on probabilistic forms of modelling to assess broad patterns and trends. However, qualitative logics of inquiry are frequently at work in computational methods too. The growingly popular technique known as topic modeling - a form of unsupervised machine learning - involves a great deal of qualitative interpretation and decision making at multiple stages of the analysis, including model fit (Bakharia et al. 2016; Isoaho et al. 2021). Quantitative and qualitative research are often described as two distinct research cultures (Mahoney and Goertz 2006). Both quantitative and qualitative research skills and logics are often required when working with computational techniques. Computational approaches to research can help bridge these cultures by productively and creatively drawing from both traditions (Bornakke and Due 2018; Brandt and Timmermans 2021; Jemielniak 2020; Mills 2018; Olson et al. 2021). The desirability of small samples for some types of research and some research questions (Benfer 1968; Small 2009) is not incompatible with computational approaches. Computational approaches can help direct researchers to compelling cases or observations that can be extracted as samples for further investigation 
and analysis using traditional qualitative techniques. Computational tools can also be used to generate and create samples from large datasets allowing researchers to zoom in on subsets for examination using qualitative techniques such as discourse analysis or content analysis (as described in greater detail in the sections that follow).

Finally, computational-qualitative inquiries draw from and contribute to theoretical discussions. Counter to early assertions that big data would usher in "the end of theory" (Anderson 2008), computational research relies on theory to guide it and is frequently a highly imaginative process (Amoore 2013). The inferences made when using computational techniques of analysis can be guided by inductive and abductive logics of inquiry (Bornakke and Due 2018; Brandt and Timmermans 2021). Given the interdependence of theoretical and empirical knowledge, as well as the many decisions about data collection, data cleaning, case sampling, and analysis involved in computational-qualitative research, reflexivity (Guillemin and Gillam 2004; Mauthner and Doucet 2003) is a core competence and should be practiced at all stages of the research process. In the sections that follow, we outline the central aspects of our proposed framework for computational-qualitative research design and provide a brief example of this approach in action.

\section{A Framework for using Computational Methods in Qualitative Research}

The framework for merging computational and qualitative approaches to data collection and analysis can be conceived in six stages: (1) defining the problem; (2) collecting; (3) parsing, exploring, and cleaning; (4) sampling and outputting; (5) analyzing; and (6) interpreting results and broader impacts (see also Baym 2013; D'Ignazio 2017). These 'stages' do not necessarily occur in the order presented and in practice can involve a great deal of back and forth. A researcher may, for example, decide to gather additional data after parsing, exploring, and cleaning what they had collected initially, a process referred to as "iteration" in qualitative research (Srivastava and Hopwood 2009). The approach that we outline below contains a mix of computational and human-driven procedures. Some of these procedures can be characterized as quantitative (e.g., counting words), others qualitative (e.g., qualitative analysis of image data). The approach is therefore best described as mixing of methods rather than purely quantitative or qualitative, although we acknowledge that there is still work to be done to address how well integrated the qualitative and quantitative components are in any blended computational-qualitative research design (Bazeley 2016).

\section{Defining the Problem}

The first step in designing a project that incorporates computational methods - as with any research project - is to determine a research question (Kraska et al. 2020; Rubin 2021). In our view, the research question should come first, although it will often evolve once the analysis has begun. With a research question in hand, methodological decisions can be made. As many methods will be appropriate, this is a pragmatic decision without any single right answer. Computational techniques will not be suitable for every research question and are not inherently superior to other means of producing knowledge. Understanding when computational techniques can be helpful versus unnecessary or hindering is important to avoid matching research questions with poorly fitted research designs and procedures. One can always incorporate a range of computational techniques into one's research design, but this 
does not mean that one should.

\section{Collecting}

There are many computational techniques available for collecting data, including web scraping (Luscombe et al. 2021; Marres and Weltevrede 2013), social media and wiki-based surveying (Bail 2017; Salganik and Levy 2015), big-data-augmented ethnography (Laaksonen et al. 2017), and online field experiments (Parigi et al. 2017; Salganik et al. 2006). Each of these methods comes with strengths and weaknesses and should be chosen in accordance with the selected research question. Web scraping, for example, is an appropriate method for collecting a large amount of text or images from a website or collection of websites, such as reports published by a government organization, posts from a social media platform like Twitter or Reddit, or articles from a news outlet.

When scraping the Internet, researchers must be sure to carefully inspect the website's terms and conditions of use as well as other relevant technical documentation (e.g., the website's robots.txt, discussed below) to make an informed legal and ethical decision about whether or not data accessible in one's web browser should be scraped (see Luscombe et al. 2021). Reviewing this documentation ahead of time is also important for algorithmic design, as many websites will ask, for example, that users moderate the speed of their requests in order not to delay server access by others. Such data, however, are all "readymades", which differs from "custommades" (Salganik 2019, 7), sometimes also referred to as "found data" and "made data" (Connelly et al. 2016; Snaphaan and Hardyns 2019). Whereas web scraping can be an effective means of collecting and repurposing readymade data available online (i.e., data not created for the purposes of research), sometimes the research question we are trying to answer warrants the collection of custommades (i.e., data that we explicitly create for the purposes of our research). To generate custom data sets, researchers need to use other kinds of computational methods, such as "crowd-sourced text analysis" (Benoit et al. 2016), which involves researchers analyzing the results of text analysis conducted by participants through online crowdsourcing, or some other existing or new method.

\section{Parsing, Exploring, and Cleaning}

Data collected via computational techniques like web scraping can be voluminous. Indeed, it is often volume that makes the use of computational methods desirable in the first place. Few people enjoy clicking through hundreds or thousands of web pages, and manually downloading material from each. Web scraping is a simple solution to this problem. Preparing data collected via computational methods for analysis typically involves three major operations. In practice, these operations take place iteratively. First, data collected using web scraping requires what is known as parsing. Parsing is an act of conversion - it involves converting one data format into another. Web scraping often involves collecting data in a format known as HTML (HyperText Markup Language). Parsing in this case involves converting raw HTML into a more human-readable format by separating the data one wants from extraneous code and information irrelevant to the research question. Another common format, often encountered by researchers obtaining data through an API (Application Programming Interface), essentially a software intermediary that allows multiple computers or computer programs to connect and interact with each other, is JSON format (JavaScript Object Notation). When 
using Twitter's API, for example, the researcher will be provided access to tweets in JSON format, which they will need to parse. There are many user-friendly tools that expedite the otherwise grueling task of parsing, such as the xml2 and httr libraries in R (Wickham et al. 2018; Wickham 2014).

Second, there is the need to explore data that have been collected. This is akin to 'getting to know' your data, which is by no means unique to the application of computational methods. In established qualitative techniques like grounded theory, data familiarization is an integral part of the research process (Charmaz 2006; Strauss and Corbin 1990). One potential point of difference is that data collected via computational methods typically appear in a digital, machine-readable format, which enables the application of certain techniques that might not be available via methods like on-site archival research or ethnographic fieldwork. ${ }^{2}$ There are many ways to conduct these initial explorations. At the highest level of generality, one could look at the quantity of data collected (such as the number of documents, the length of each document in words, sentences, or paragraphs) or the format of the data collected (e.g., written documents vs images). One might also familiarize them self with the content of the data collected, immersing oneself, to better understand what it contains and what it does not, such as by examining a small sample.

Finally, computationally harvested data almost always requires cleaning. When working with large quantities of data especially, cleaning can take a significant amount of effort. According to one oft-stated estimate, data cleaning can take up to $80 \%$ of the total effort that goes into a research project (Dasu and Johnson 2003). Cleaning takes many forms. One may, for example, need to convert non-searchable (but still digital) text data (like pdf files obtained through Access to Information, Freedom of Information, or public records requests) into a machinereadable format using Optical Character Recognition (OCR). Although the exact accuracy will depend on the OCR engine one is using, the results are imperfect. At the very least, all or some sample of the output of OCR should be checked for quality control. Other common cleaning operations include fixing typographical errors, case folding (the process of converting text to lower or upper case), removal of punctuation, numbers, stop words (extremely common words that tend to have little analytical value like "the"), and other unwanted characters, stripping extra whitespace, and word stemming and lemmatization (reducing words to their root).

\section{Sampling and Outputting}

Often social researchers will have access to more information than one can reasonably handle with a 'thick' qualitative analysis. In such situations, it is prudent to construct a sample. Sampling is a decision about what portions of a larger data set (usually called a 'population' in statistics) to include in a study, answering the questions of how and why a particular number of cases are selected for inclusion (Gentles et al. 2015). One way that purposive sampling can be achieved is via a combination of string-searching and filtering algorithms. For example, we might only want documents or paragraphs within a set of documents that contain a predetermined list or 'dictionary' of words. We refer to this as dictionary-based purposive

\footnotetext{
${ }^{2}$ This distinction dissipates when one considers that archival files and observational field notes too can both easily be digitized and rendered into a machine-readable format. The difference, then, is more a matter of convenience than anything; data collected via techniques like web scraping "live" on one's computer, making it very easy to jump straight into many of the computer-driven familiarization techniques that we discuss.
} 
sampling. Alternatively, we might want all texts that correspond to a certain period, place, or other piece of metadata we collected. A final technique of computationally assisted purposive sampling we will mention is more quantitative. Here the researcher calculates some metric, such as Term Frequency-Inverse Document Frequency or "TF-IDF" (Jones 1972), a popular metric in information science for determining "important words" in text, and samples on this measure. $^{3}$ A more sophisticated approach would be to use something like latent Dirichlet allocation (a popular algorithm for the machine learning approach known as topic modeling) to generate topics from the corpus and sample on the basis of these.

A final strategy we note would be to randomly sample a subset of data from the corpus. This can be achieved several ways. For example, one might choose to construct a 'simple random sample' by grabbing a predetermined number of cases or a percentage of cases. Or one might use a more sophisticated random sampling technique like 'stratified random sampling' to construct a sample of texts partitioned along some observed feature of the corpus, such as time or location. Although random sampling can be an appropriate strategy for selecting cases for qualitative analysis, particularly when one is beginning from a large dataset, random sampling should be used only when appropriate, as random sampling is not always suited to the goals of qualitative research (Benfer 1968; Small 2009).

\section{Analyzing and Interpreting}

How one analyzes their data will depend on the research question as well as the role of theory in the research design. Although it is beyond the scope of this paper to discuss strategies of qualitative analysis in any detail, some options include grounded theory (Charmaz 2006), abductive analysis (Timmermans and Tavory 2012), critical discourse analysis (Fairclough 2013), semiotic analysis (Cullum-Swan and Manning 1994), and metaphor analysis (Schmitt 2005). Below, we demonstrate how computational methods can assist with visual analysis through an analysis of images included in RCMP news releases.

Our analysis is intended to demonstrate how computational methods can help to orient qualitative research and offers early-stage findings and connections that social researchers might build upon to produce research for publication. Our analysis serves as an example for purposes of criminology and criminal justice pedagogy and only gestures to potential research trajectories that might be pursued in a fuller analysis. However, it is important to note that the interpretation of findings and engagement with their implications for academic knowledge and broader social context are crucial steps in producing research for publication in the social sciences. At this point in this article, the reader is encouraged to consult the supplement, which can be read before, alongside, or after the case study of RCMP news releases presented next.

\footnotetext{
${ }^{3}$ To calculate a TF-IDF score, the researcher first calculates the term frequency of a given word (the TF in TF-IDF), that is, how many times the word appears across a set of documents (or other unit of analysis, e.g., paragraphs). Next, the researcher calculates the inverse document frequency of the word (the IDF in TF-IDF). This is obtained by taking the log of the result of the total number of documents divided by the total number of documents containing the word of interest. Multiplying these two numbers together (the TF and the IDF) generates a TF-IDF score for the word. The larger the TF-IDF score, the more important the word is in a given document.
} 


\section{A Case Study of RCMP News Releases}

\section{Defining the Problem}

Media representations of crime have attracted scholarly attention for some time, however, the popularity of methods like qualitative content and discourse analysis alongside the relative ease of access to textual data has resulted in a slant toward written discourse in much of the existing work (Bucholtz 2001). Studies that extend their attention to multi-modal representations of policing and criminal justice often still take texts as their starting point (Duncan and Walby 2021). Despite this apparent bias in social sciences research there has been a rise in the use of visual methods in criminal justice research in recent years (Carrabine 2015). Our research question is as follows: how do the Royal Canadian Mounted Police (RCMP) visually represent their policing work in Canada? More specifically, what 'work' do the images included in RCMP press releases do with respect to conveying a message about policing and social control?

There are a growing number of examples of visual methods applied to the study of policing. Linnemann (2017) has written about how "visual economies" of policing work to demonstrate their skill as hunters through images of seized cash, drugs and weapons - a practice that mirrors displays of wealth popularly associated with narco-traffickers and street gangs. Wall and Linnemann (2014) have discussed how mugshot images are used to craft sensationalist narratives about methamphetamine users in the United States linking them to the broader cultural trope of 'white trash'. Lippert and Wilkinson (2010) analyze how the collection and distribution of images captured by private closed-circuit television (CCTV) cameras and shared by the Crime Stoppers 'tip line' elevates the perceived prevalence and importance of crimes that impact businesses at the expense of vulnerable people. Walby and Wilkinson (2021) show how Canadian police highlight images of diversity and community engagement on Instagram in efforts to bolster institutional legitimacy. As these studies demonstrate, visual criminologists obtain their data from a variety of sources that are usually publicly accessible. Our analysis focuses on images attached to news releases produced by the RCMP. We drew only secondarily on the text of the news releases when additional context was necessary.

\section{Collecting}

To collect our data, we used web scraping to automatically extract text and image data from the news section of the RCMP's website. Prior to conducting our scrape, we inspected the website's terms and conditions of use as well as the instructions contained in the website's 'robots exclusion protocol' or robots.txt, which can be accessed by simply adding / robots.txt to any website's root directory. Guided by what we call "algorithmic thinking in the public interest" (Luscombe et al. 2021), we used this documentation to make an informed legal and ethical decision about whether our scrape was fair and justified. In accordance with the RCMP's robots.txt directives, we added a 20-second crawl delay into our web scraping script, meaning that our bot paused 20-seconds before sequencing along to the next page in the RCMP's news portal.

There are many ways to scrape the web. For this study, we conducted our scrape in R (an open-source programming language) using a library (a collection of community-developed functions) called rvest (Wickham 2016). The scrape unfolds in two phases. We refer to 
the first as the "index scrape", and the second as the "contents scrape". The index scrape works by automatically 'clicking' through the page elements containing links to each of the RCMP's news releases, extracting link information for each individual news release, as well other metadata that is available (like the title of the news release). In this first scrape, our primary goal was to obtain each of these links, building an index of news releases. Next, we build the contents scrape that visits each of the links in the index to retrieve information from each of the pages. From each of the page links, we obtained the title of the news release, the date of the news release, the location of the RCMP agency submitting the news release, the written contents of the news release, and a link to any images contained in the news release. ${ }^{4}$

\section{Parsing, Exploring, and Cleaning}

In 'getting to know' our data, we asked many questions, which were answered primarily using data manipulation and visualization capabilities available in R's ggplot2 and dplyr libraries (Wickham et al. 2016, 2015). The first set of questions we asked pertained to the distribution of our cases across time and space. Which provinces and territories accounted for the majority news in our corpus, and how were these cases distributed across provinces and territories over time? In answering these questions, we made several discoveries. First, we discovered the location information obtained from our scrape was messy and would need to be cleaned up before we could proceed. For certain entries, only town, city, and/or county had been provided, requiring us to manually enter the name of the province and territory for each, which we achieved by writing a function in $\mathrm{R}$.

After properly tagging location in each entry, we were able to plot the distributions of news over time and across regions. This led us to our next two discoveries. First, we discovered that news from the province of British Columbia was massively underrepresented in our corpus (Figure 1, Panel A). Upon further investigation, we learned the reason for this was that the BC RCMP has its own digital news release portal, which we could not subsequently scrape as entries appear to be deleted from the portal on a rolling basis. At the time of writing, the available RCMP news release data ended May 5, 2021. Second, we discovered regional differences in the frequency of RCMP news by province/territory over time (Figure 1, Panels $\mathrm{B}$ and $\mathrm{C}$ ). Although we do not examine these regional differences closely in our illustration below, these would certainly be important to consider in any larger scale, in-depth analysis of the corpus.

The next set of questions we asked pertained to the structure and content of the news entries themselves. First, we wanted to know how long RCMP news entries were on average, across the entire corpus and by province/territory (Figure 1, Panel D). To do this, we counted the number of words in each press release and plotted the results as a separate density plot (a smoothed variation of the histogram plot) for each province/territory. Finally, as our research questions pertained to the visual component of the corpus, we wanted to know how many of the news releases contained links to photos (Table 1).

\footnotetext{
${ }^{4}$ The index and contents scrape can be thought of like a Google search. When searching the word 'crime' on Google, one arrives first at a page containing various links to other websites. This first page (and subsequent pages) can be thought of as comprising the 'index'; it contains the links to the pages we may (or may not be) interested in visiting. Clicking on any given link in a Google result brings us to the website itself. The material on this website can be thought of as the contents, which would be obtained in the second ('contents') scrape.
} 
A

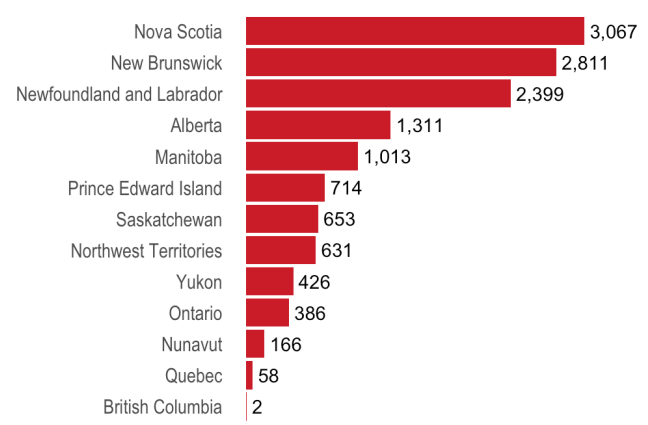

C

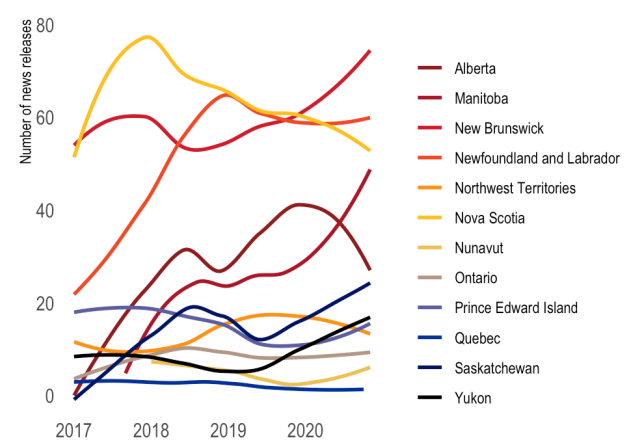

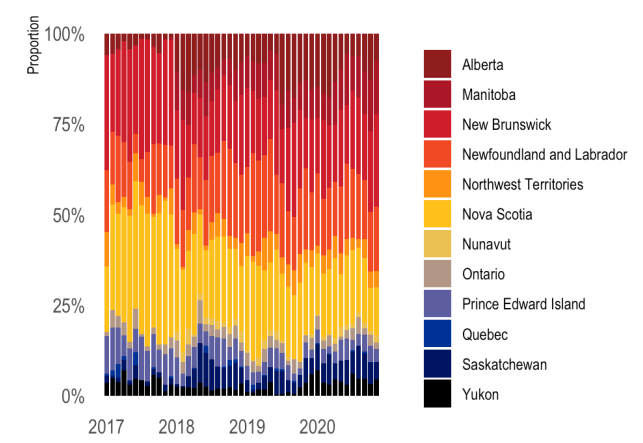

D

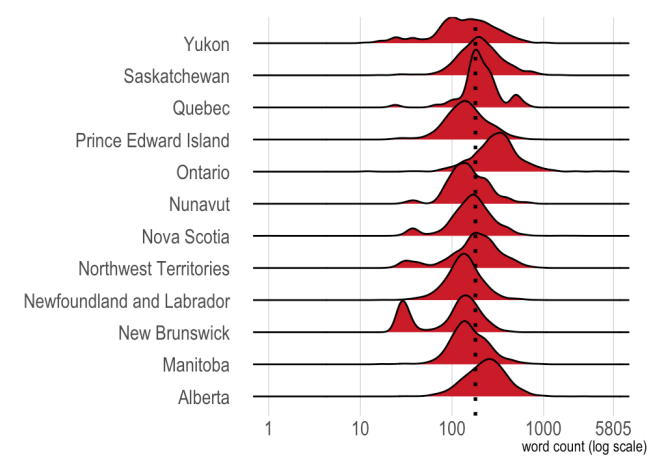

Figure 1: Panel A: Total number of RCMP press releases by province/territory. Includes only news releases available at the time we conducted the scrape, covering the period 201612-07 to 2020-11-27. Panel B: Total proportion of RCMP press releases over time, by province/territory, excluding British Columbia. Proportion reflects the total percentage of monthly news releases posted by RCMP in each province/territory. Panel C: Smoothed frequency plot of news release distribution over time by province/territory by number of releases. Panel D: Density plot distribution of total word count in RCMP press releases. The x-axis is in $\log$ scale. Dotted line indicates the whole corpus mean word count of $n=179$. The extreme ends of the $\mathrm{x}$-axis in this graph show the range of minimum and maximum values. The shortest news release in the corpus had a word count of 1 (Nova Scotia). The longest news release in the corpus had a word count of 5,805 (Ontario). 


\begin{tabular}{l|r|r|r}
\hline Province/Territory & Total & Images & Percent \\
\hline Nunavut & 166 & 155 & 93.37 \\
\hline Nova Scotia & 3067 & 2612 & 85.16 \\
\hline New Brunswick & 2811 & 2360 & 83.96 \\
\hline Northwest Territories & 631 & 495 & 78.45 \\
\hline Prince Edward Island & 714 & 550 & 77.03 \\
\hline Newfoundland and Labrador & 2399 & 1834 & 76.45 \\
\hline Alberta & 1311 & 978 & 74.60 \\
\hline Manitoba & 1013 & 704 & 69.50 \\
\hline Yukon & 426 & 275 & 64.55 \\
\hline Ontario & 386 & 237 & 61.40 \\
\hline Quebec & 58 & 27 & 46.55 \\
\hline Saskatchewan & 653 & 88 & 13.48 \\
\hline
\end{tabular}

Table 1: Total news releases in corpus by province/territory, excluding British Columbia. Total column indicates the total number of news releases (with and without images), images and percent columns indicate the total number and percentage of news releases containing at least one image. Darker colours and lighter fonts indicate larger numbers. 


\section{Sampling and Outputting}

To sample our data, we used stratified random sampling. We first created a sample of all news releases that contained images ( $\mathrm{N}=10,315$, or $76 \%$ of the total corpus). From this larger sample we constructed a stratified random sample for analysis, taking $15 \%$ of all news releases treating year and province/territory as strata. This sampling strategy ensured that we did not over-sample from select time periods or regions, allowing us to retain some of the temporal and geographic richness and variation of the larger data set. This gave us a final sample of 497 unique RCMP news releases containing images. Next, we wrote a script to automatically download the images in our sampled corpus and store them on an external hard drive, named by province/territory, date of the news release, plus a unique identifier that we created. We also downloaded the textual content for each of the corresponding news releases, specifically title, date, city/town, province, and the main text of the news release itself, although we make only limited use of it in this illustration. We named these files to match the images and stored them in the same folder so that the two could be easily analyzed together.

\section{Analyzing}

To analyze our sampled images, we used NVivo 12, a qualitative research software that facilitates inquiry by allowing researchers to organize content into categories or 'codes' and take notes. Borrowing from grounded theory, we adopted an 'open coding' (Strauss and Corbin 1990) approach to the images and placed them in 13 different high-level categories (see Table 1 below). Of the images sampled, 67 were logos attached to news releases posted by the Saskatchewan RCMP and were coded as such but are omitted from this analysis. In a larger-scale analysis, one might apply techniques from computer vision (see e.g., Khorshidi et al. 2021) to automatically identify and filter out duplicate images such as these to save time. However, automation cannot replace the process of reckoning (Smith 2019) that goes into sorting the images and making meaning from the categories as we have opted to do in this analysis. We chose a best fit approach for our coding meaning each image was assigned only one category, even if there was another category that it might have fit into. For example, images of a car wreck were coded as "Damage \& Destruction" even though there was a vehicle in the image. In larger research projects, the open coding stage is typically followed by axial and selective coding stages where top-level codes are re-organized and refined with 'child nodes' or sub-codes that break down and re-organize the general categories. For example, under "Identification" one might sub-divide the images into those captured by CCTV cameras, mugshots, police sketches, and personal photos. For most of the images, their purpose was self-evident, however, in some cases it was necessary to consult the text file corresponding to an image for additional context. For example, some of the images in the 'Objects of Interest' code required this type of secondary inquiry, whereas for the 'Spoils of War' code, the purpose of the images was typically clear.

\section{Interpreting the Findings}

As shown in Table 2, there are 13 top-level codes that were used to sort and analyze the images attached to RCMP press releases. In this section, we first describe the four most common in some detail and offer more general descriptions of the remaining nine. Following 


\begin{tabular}{llr} 
Code & Description & References \\
\hline Identification & Images of people for the purposes of identifying them & 98 \\
\hline Spoils of War & Seized money, drugs, weapons, or other goods & 93 \\
\hline Vehicle & Cars, airplanes, off-road vehicles, bikes, etc. & 63 \\
\hline Line of Duty & Depictions of police work & 37 \\
\hline Damage \& Destruction & Damaged property including buildings and vehicles & 30 \\
\hline Community Engagement & Depictions of positive interactions with community & 21 \\
\hline Objects of Interest & Objects that may have been stolen or are serving as evidence & 17 \\
\hline PSA & Public Service Announcements (edited images) & 17 \\
\hline Clipart \& Stock Images & Generic stock images or animated clipart & 15 \\
\hline Rank and File & Ceremony and militaristic formalism & 15 \\
\hline Misc. & Do not fit into any clear category & 11 \\
\hline Animals & Police service animals and wild animals & 9 \\
\hline Maps & Maps depicting areas of interest to police & 4 \\
\hline
\end{tabular}

Table 2: Total number of references of each code in the sample $(\mathrm{N}=497)$. Code column indicates code label, description column provides a brief description of the code, and the references column indicates total number of images representing this code.

this we discuss how our exploratory findings connect to existing literature and how more in-depth analyses might be undertaken to produce novel insights. In the article supplement, we provide example images for each of the first four codes.

The most published images in the data are those seeking to identify an individual. There are several types of identification images that a researcher could organize into subcodes for further analysis. These include CCTV images, mugshots, forensic sketches, and personal photos, which support police in efforts to identify and locate suspects and missing people.

The second most common type of image was of items seized by police, which we refer to as 'spoils of war'. Seizures varied in terms of the contents, scale, and presentation. Contents of these seizures include weapons, drugs and drug paraphernalia, cash, motorcycle club merchandise, alcohol, and tobacco products. The scale of seizures ranges from a single item like a handgun to many kilos of narcotics. All the images are to some extent staged, which is to say they are arranged to convey a message. The exemplary spoils of war image is taken from above a table with items arranged in an orderly grid with cash spread to emphasize its value; often a sign or table cloth with the RCMP or division insignia is used to claim ownership of the goods in the photograph. Other times, seizures are photographed in situ to emphasize their scale, or the crafty ways illicit goods were hidden, thus highlighting the exceptional skill of the officers who seized them. In some cases, the orderly grid aesthetic is achieved by making photo collages of individual items. Lastly, sometimes the exception to the norm can be just as interesting as the broader pattern as with one image in which an officer proudly poses with two seized handguns.

Third most common are images of vehicles including cars, bicycles, off-road vehicles, snowmobiles, and even an airplane. Of all the codes, the images of vehicles tended to tell the least 
clear story on their own, so we consulted some of the news releases associated with them for additional context. Typically, images from CCTV cameras, stock product photos, and personal photos shared with police are used to help identity and locate stolen vehicles. Higher quality photos of vehicles are sometimes shared to show off the recovery of stolen property. Finally, and less commonly, images of police vehicles including cars, a boat, an off-road utility vehicle, and a helicopter are shared and often staged in ways that celebrate and emphasize the exceptional mobility of police.

The fourth most common code is titled 'line of duty' and refers to images depicting police work in action. Within these images are two primary sub themes. The first refers to perspectival images, where a photo is taken with the intent of encouraging the viewer to 'see like the police', whether from the dash of their patrol car, over a speed radar gun, and in one case from an aerial vehicle looking down. The second category are more stylized depictions of police work, most commonly shot down a road at an angle capturing police vehicles and the subject of their interest (for example, a truck overturned on the road) although there are also staged shots of officers in a park, performing an emergency operation in the snow, and a portrait of an officer next to their vehicle. Within the 'line of duty' code there are many images that do not conform to these two aesthetic trends, including an aerial shot of a car-chase, a picture of an office setting, a training seminar, and a collage (in the same grid style as found in the spoils of war code) depicting police work as involving action, training, and technical computer skills.

Apart from the four codes highlighted above, the other nine also revealed interesting trends in police communication. The damage and destruction code contained images of destroyed buildings and car wrecks. Images depicting community engagement work to emphasize positive engagement with community members and to soften the image of police presented in the 'line of duty' images, sometimes through dark humour such as letting kids handcuff each other or putting the Grinch in the backseat of a patrol car. The police also shared images of objects of interest including pictures of evidence and stolen items. Public Service Announcements were largely computer generated and edited images promoting individual responsibility from crime prevention like locking up one's house and car properly, avoiding internet scams, and staying safe on Halloween. Many of the images attached to news releases were animated clip art or generic stock photos. The rank-and-file code included images that featured a militaristic or ceremonial aesthetic often denoted by the RCMP's characteristic bright red formal uniform. A miscellaneous code captured images that did not fit neatly in another category such as an image of a traffic cone, a newly opened detachment building, and an image of a river. Images of animals featured rarely; most often these images depicted service animals, however one picture of three black bears was included in this code as well. The least common code was for images of maps depicting areas of interest to police.

Based on this inquiry, there are several trends that a researcher could examine further. We can observe many similar trends as already exist in the literature. With respect to police depictions of criminality, one might extend Wall and Linnemann's (2014) analysis of mugshots through a systematic race- and gender-based analysis of the mugshots shared by the RCMP. Similarly, Lippert and Wilkinson's (2010) insights regarding CCTV camera footage might be added to by unpacking how the 'raw' aesthetic of surveillance footage contributes to certain narratives about the seriousness and prevalence of certain types of criminality. The images also do work to convey ideas about policing itself. One could build further on Linnemann's (2017) analysis of American police seizures by asking what the RCMP's use of spoils of war 
images tells us about their strategic narrative goals and how they wish to portray police work. Additionally, the images of community engagement could help to extend Walby and Wilkinson's (2021) discussion of how police use Instagram.

Further to the above examples of existing visual criminological studies that a researcher might seek to build upon, there are endless possibilities to engage in dialogues that have yet to fully enter the domain of visual criminology. Here we highlight two. First, researchers may wish to connect the RCMP's use of images in their news releases with academic discussions of mobility. It is clear, even at the high level of coding, that the images of police vehicles sought to glamorize police mobility whether showing off vehicles that everyday people might not have access to like helicopters or staging pictures of cruisers to make them look hip or cool. Secondly, we found the stylized depictions of police work in the line of duty code to be both visually compelling and cohesive. What is achieved by the recurring visual trope of a cruiser shot at an angle down the road? What might this reveal about how the RCMP wishes to depict police work? The politics of these representations can be further examined, and computational approaches to research can assist in this endeavour in many ways.

\section{Discussion and Conclusion}

Criminologists and criminal justice researchers - including those operating in predominantly qualitative traditions - stand to benefit by leveraging technically mediated access to vast and variegated quantities of digital information. This requires incorporating critical algorithmic thinking (Green and Viljoen 2020) into research design and research questions; at the same time, we have claimed it would be wrong to associate computational approaches to research strictly with quantitative methods and research design. Beyond the implications of big data for criminal justice practices, developments in computational science have major implications for methodology in criminology and criminal justice studies. Embracing the computational turn in criminology does not require rejecting traditional qualitative and quantitative methods. We have shown that computational approaches to criminology and criminal justice studies draw from and extend beyond traditional distinctions between qualitative and quantitative methods. We have demonstrated how qualitative logics of inquiry and skill sets can be folded into computational research. We have demonstrated this by showing some of the iterative steps involved in using computational approaches to examine police communications in ways that cannot be fully automated, and which complement the 'thinner' insights of quantitative methods with the 'thicker' insights of qualitative ones (Bornakke and Due 2018; Jemielniak 2020; Olson et al. 2021).

In the computationally driven process of data collection and case sampling we have used in this article, the larger corpus and subset are never entirely divorced, a feature with implications for several traditional qualitative notions and techniques including iteration and reflexivity. Computational methods like web scraping and techniques of purposive and random sampling afford the researcher the ability to sample and re-sample from the same corpus as many times as necessary. This attribute of digital corpora has implications for notions like "transferability" (Tracy 2010). Although we did not do this in our case study illustration, it is possible to add a final, deductive stage when using this kind of research design, where the researcher assesses the transferability of their findings at the subset corpus level on the whole corpus (e.g., Nelson 2020).

We have explained how a computational approach enables the researcher to collect and analyze 
a large corpus of visual data in ways that would not be possible - or would at least be more labour intensive - if one started with a more conventional qualitative approach. We have further sought to reveal how a multi-modal qualitative approach to discourse and image analysis enables the researcher to dig deeper into the layered meanings contained in data than most computational techniques can alone. Our analysis of RCMP image data offers a single illustration of how to combine computational and qualitative criminology in ways that are mutually enriching and reinforcing. The online supplement to this paper serves as a guide for researchers interested in building their methodological skillset or expanding their approach to teaching research methods in the social sciences as well.

The adoption of computational methods in criminology and criminal justice studies could help in responding to the "coming crisis" in social science research (Gouldner 1972; Savage and Burrows 2007) and associated concerns about methodological and pedagogical stagnation, trustworthiness, transparency, and credibility in the social sciences. The methodological steps, decisions, and code provided in this article and its supplement are all open, transparent, and available for interrogation and replication. In doing so, we hope to have showcased an approach to methodology that will allow criminology and criminal justice studies to gain from advances being made in the computer and information sciences while at the same time staying true to the longer legacy of qualitative inquiry in sociology, anthropology, and other cognate disciplines.

\section{References}

Al-Zaidy, R., Fung, B. C., Youssef, A. M., and Fortin, F. (2012). Mining criminal networks from unstructured text documents. Digital Investigation, 8(3-4):147-160.

Amoore, L. (2013). The politics of possibility. Duke University Press.

Anderson, C. (2008). The end of theory: The data deluge makes the scientific method obsolete. Wired magazine, 16(7):16-07.

Back, L. (2012). Live sociology: social research and its futures. The Sociological Review, $60: 18-39$.

Bail, C. A. (2017). Taming big data: Using app technology to study organizational behavior on social media. Sociological Methods \& Research, 46(2):189-217.

Bakharia, A., Bruza, P., Watters, J., Narayan, B., and Sitbon, L. (2016). Interactive topic modeling for aiding qualitative content analysis. In Proceedings of the 2016 ACM on Conference on Human Information Interaction and Retrieval, pages 213-222.

Baym, N. K. (2013). Data not seen: The uses and shortcomings of social media metrics. First Monday.

Bazeley, P. (2016). Mixed or merged? integration as the real challenge for mixed methods. Qualitative Research in Organizations and Management: An International Journal, 11:189194.

Benfer, R. A. (1968). The desirability of small samples for anthropological inference. American Anthropologist, 70(5):949-951. 
Benoit, K., Conway, D., Lauderdale, B. E., Laver, M., and Mikhaylov, S. (2016). Crowdsourced text analysis: Reproducible and agile production of political data. American Political Science Review, 110(2):278-295.

Bornakke, T. and Due, B. L. (2018). Big-thick blending: A method for mixing analytical insights from big and thick data sources. Big Data 85 Society, 5(1).

Brandt, P. and Timmermans, S. (2021). Ab-ductive logic of inquiry for quan-titative research in the digital age. Sociological Science, 8:191-210.

Brayne, S. (2017). Big data surveillance: The case of policing. American sociological review, 82(5):977-1008.

Bucholtz, M. (2001). Reflexivity and critique in discourse analysis. Critique of anthropology, 21(2):165-183.

Carrabine, E. (2015). Visual criminology: history, theory, and method. In The Routledge handbook of qualitative criminology, pages 119-138. Routledge.

Chan, J. and Bennett Moses, L. (2016). Is big data challenging criminology? Theoretical criminology, 20(1):21-39.

Charmaz, K. (2006). Constructing grounded theory: A practical guide through qualitative analysis. sage.

Cheng, T. (2021). Social media, socialization, and pursuing legitimation of police violence. Criminology, 59(3):391-418.

Collier, D., Brady, H. E., and Seawright, J. (2004). Sources of leverage in causal inference: Toward an alternative view of methodology. In Collier, D. and Brady, H. E., editors, Rethinking social inquiry: Diverse tools, shared standards, pages 229-266. Rowman \& Littlefield Lanham, MD.

Connelly, R., Playford, C. J., Gayle, V., and Dibben, C. (2016). The role of administrative data in the big data revolution in social science research. Social science research, 59:1-12.

Cullum-Swan, B. and Manning, P. (1994). Narrative, content, and semiotic analysis. Handbook of qualitative research, pages 463-477.

Dasu, T. and Johnson, T. (2003). Exploratory data mining and data cleaning, volume 479. John Wiley \& Sons.

D'Ignazio, C. (2017). Creative data literacy: Bridging the gap between the data-haves and data-have nots. Information Design Journal, 23(1):6-18.

Duncan, J. and Walby, K. (2021). Police union political communications in canada. The British Journal of Criminology, Online-First.

Fairclough, N. (2013). Critical discourse analysis: The critical study of language. Routledge.

Ferguson, A. G. (2017). The rise of big data policing. New York University Press. 
Gentles, S. J., Charles, C., Ploeg, J., McKibbon, K. A., et al. (2015). Sampling in qualitative research: Insights from an overview of the methods literature. The qualitative report, 20(11):1772-1789.

Gouldner, A. W. (1972). The coming crisis of western sociology. Basic Books.

Green, B. and Viljoen, S. (2020). Algorithmic realism: expanding the boundaries of algorithmic thought. In Proceedings of the 2020 Conference on Fairness, Accountability, and Transparency, pages 19-31.

Guillemin, M. and Gillam, L. (2004). Ethics, reflexivity, and "ethically important moments" in research. Qualitative inquiry, 10(2):261-280.

Halford, S. and Savage, M. (2017). Speaking sociologically with big data: Symphonic social science and the future for big data research. Sociology, 51(6):1132-1148.

Hannah-Moffat, K. (2019). Algorithmic risk governance: Big data analytics, race and information activism in criminal justice debates. Theoretical Criminology, 23(4):453-470.

Isoaho, K., Gritsenko, D., and Mäkelä, E. (2021). Topic modeling and text analysis for qualitative policy research. Policy Studies Journal, 49(1):300-324.

Jackson, K. and Bazeley, P. (2019). Qualitative data analysis with NVivo. Sage.

Jemielniak, D. (2020). Thick big data: Doing digital social sciences. Oxford University Press.

Joh, E. E. (2016). The new surveillance discretion: automated suspicion, big data, and policing. Harv. L. \& Pol'y Rev., 10:15.

Jones, K. S. (1972). A statistical interpretation of term specificity and its application in retrieval. Journal of documentation.

Khorshidi, S., Carter, J., Mohler, G., and Tita, G. (2021). Explaining crime diversity with google street view. Journal of Quantitative Criminology, 37(2):361-391.

Kitchin, R. (2014). Big data, new epistemologies and paradigm shifts. Big data 8 society, 1(1):2053951714528481.

Kleck, G., Tark, J., and Bellows, J. J. (2006). What methods are most frequently used in research in criminology and criminal justice? Journal of Criminal Justice, 34(2):147-152.

Kraska, P. B., Brent, J. J., and Neuman, W. L. (2020). Criminal justice and criminology research methods. Routledge.

Kuang, D., Brantingham, P. J., and Bertozzi, A. L. (2017). Crime topic modeling. Crime Science, 6(1):1-20.

Laaksonen, S.-M., Nelimarkka, M., Tuokko, M., Marttila, M., Kekkonen, A., and Villi, M. (2017). Working the fields of big data: Using big-data-augmented online ethnography to study candidate-candidate interaction at election time. Journal of Information Technology ES Politics, 14(2):110-131. 
Linnemann, T. (2017). Proof of death: Police power and the visual economies of seizure, accumulation and trophy. Theoretical Criminology, 21(1):57-77.

Lippert, R. and Wilkinson, B. (2010). Capturing crime, criminals and the public's imagination: Assembling crime stoppers and cctv surveillance. Crime, media, culture, 6(2):131-152.

Lippert, R. K. and Molnar, A. (forthcoming). Surveillance, police, and quarantining covid-19 in canada and australia. In Hamilton, C. and Nelken, D., editors, Research Handbook on Comparative Criminal Justice. Edward Elgar.

Locke, K., Golden-Biddle, K., and Feldman, M. S. (2008). Perspective - making doubt generative: Rethinking the role of doubt in the research process. Organization science, 19(6):907918.

Luscombe, A., Dick, K., and Walby, K. (2021). Algorithmic thinking in the public interest: navigating technical, legal, and ethical hurdles to web scraping in the social sciences. Quality 8 Quantity, pages 1-22.

Mahoney, J. and Goertz, G. (2006). A tale of two cultures: Contrasting quantitative and qualitative research. Political analysis, 14(3):227-249.

Marres, N. and Weltevrede, E. (2013). Scraping the social? issues in live social research. Journal of cultural economy, 6(3):313-335.

Mauthner, N. S. and Doucet, A. (2003). Reflexive accounts and accounts of reflexivity in qualitative data analysis. Sociology, 37(3):413-431.

McAfee, A., Brynjolfsson, E., Davenport, T. H., Patil, D., and Barton, D. (2012). Big data: the management revolution. Harvard business review, 90(10):60-68.

McKie, L. and Ryan, L. (2015). An end to the crisis of empirical sociology?: trends and challenges in social research. Routledge.

Mills, K. A. (2018). What are the threats and potentials of big data for qualitative research? Qualitative Research, 18(6):591-603.

Nelson, L. K. (2020). Computational grounded theory: A methodological framework. Sociological Methods \&6 Research, 49(1):3-42.

Olson, A. W., Calderon-Figueroa, F., Bidian, O., Silver, D., and Sanner, S. (2021). Reading the city through its neighbourhoods: Deep text embeddings of yelp reviews as a basis for determining similarity and change. Cities, 110:103045.

Parigi, P., Santana, J. J., and Cook, K. S. (2017). Online field experiments: studying social interactions in context. Social Psychology Quarterly, 80(1):1-19.

Pina-Sánchez, J., Grech, D., Brunton-Smith, I., and Sferopoulos, D. (2019a). Exploring the origin of sentencing disparities in the crown court: Using text mining techniques to differentiate between court and judge disparities. Social science research, 84:102343.

Pina-Sánchez, J., Roberts, J. V., and Sferopoulos, D. (2019b). Does the crown court discriminate against muslim-named offenders? a novel investigation based on text mining techniques. The British Journal of Criminology, 59(3):718-736. 
Rubin, A. T. (2021). Rocking Qualitative Social Science: An Irreverent Guide to Rigorous Research. Stanford University Press.

Salganik, M. J. (2019). Bit by bit: Social research in the digital age. Princeton University Press.

Salganik, M. J., Dodds, P. S., and Watts, D. J. (2006). Experimental study of inequality and unpredictability in an artificial cultural market. science, 311(5762):854-856.

Salganik, M. J. and Levy, K. E. (2015). Wiki surveys: Open and quantifiable social data collection. PloS one, 10(5):e0123483.

Sandberg, J. and Alvesson, M. (2011). Ways of constructing research questions: gap-spotting or problematization? Organization, 18(1):23-44.

Sanders, C. B. and Sheptycki, J. (2017). Policing, crime and 'big data'; towards a critique of the moral economy of stochastic governance. Crime, law and social change, 68(1):1-15.

Savage, M. (2015). Sociology and the digital challenge. Innovations in digital research methods, pages $297-310$.

Savage, M. and Burrows, R. (2007). The coming crisis of empirical sociology. Sociology, $41(5): 885-899$.

Schmitt, R. (2005). Systematic metaphor analysis as a method of qualitative research. The qualitative report, 10(2):358-394.

Small, M. L. (2009). How many cases do i need?' on science and the logic of case selection in field-based research. Ethnography, 10(1):5-38.

Smith, B. C. (2019). The promise of artificial intelligence: reckoning and judgment. Mit Press.

Smith, G. J., Bennett Moses, L., and Chan, J. (2017). The challenges of doing criminology in the big data era: Towards a digital and data-driven approach. The British journal of criminology, 57(2):259-274.

Snaphaan, T. and Hardyns, W. (2019). Environmental criminology in the big data era. European Journal of Criminology, Online-First.

Srivastava, P. and Hopwood, N. (2009). A practical iterative framework for qualitative data analysis. International journal of qualitative methods, 8(1):76-84.

Strauss, A. and Corbin, J. (1990). Basics of qualitative research. Sage publications.

Thomson, R. and Holland, J. (2003). Hindsight, foresight and insight: The challenges of longitudinal qualitative research. International Journal of Social Research Methodology, 6(3):233-244.

Timmermans, S. and Tavory, I. (2012). Theory construction in qualitative research: From grounded theory to abductive analysis. Sociological theory, 30(3):167-186. 
Tracy, S. J. (2010). Qualitative quality: Eight "big-tent" criteria for excellent qualitative research. Qualitative inquiry, 16(10):837-851.

Travers, M. (2009). New methods, old problems: A sceptical view of innovation in qualitative research. Qualitative research, 9(2):161-179.

Vander Beken, T., Vandeviver, C., and Daenekindt, S. (2021). Two decades of european criminology: Exploring the conferences of the european society of criminology through topic modelling. European Journal of Criminology, page 14773708211007384.

Walby, K. and Wilkinson, B. (2021). The visual politics of public police instagram use in canada. New Media $\&$ Society, Online-First.

Wall, T. and Linnemann, T. (2014). Staring down the state: Police power, visual economies, and the "war on cameras". Crime, Media, Culture, 10(2):133-149.

Wickham, H. (2014). httr: Tools for working with urls and http. $R$ package version 0.5 , URL http://CRAN. R-project. org/package $=$ httr.

Wickham, H. (2016). rvest: Easily harvest (scrape) web pages. R package version 0.3, 2:2016.

Wickham, H., Chang, W., Henry, L., Pedersen, T. L., Takahashi, K., Wilke, C., Woo, K., Yutani, H., Dunnington, D., et al. (2016). ggplot2: Create elegant data visualisations using the grammar of graphics. $R$ package version, $2(1)$.

Wickham, H., Francois, R., Henry, L., Müller, K., et al. (2015). dplyr: A grammar of data manipulation. $R$ package version 0.4, 3:p156.

Wickham, H., Hester, J., and Ooms, J. (2018). xml2: Parse xml. R package version, 1(0).

Williams, M. L. and Burnap, P. (2016). Cyberhate on social media in the aftermath of woolwich: A case study in computational criminology and big data. British Journal of Criminology, 56(2):211-238.

\section{Affiliation:}

Alex Luscombe

Centre for Criminology and Sociolegal Studies

University of Toronto

Toronto, Ontario

E-mail: alex.luscombe@mail.utoronto.ca

URL: www .alexluscombe.ca

\section{SocArXiv Website SocArXiv Preprints}

Journal of Criminal Justice Education

doi.org/10.1080/10511253.2022.2027477 https://socopen.org/

https://osf.io/preprints/socarxiv

Submitted: August 30, 2021 Accepted: November 29, 2021 